\title{
Virginia Apgar y la anestesiología obstétrica. Aportes, anécdotas y aficiones
}

\author{
Alfredo Jácome Roca, MD, FACP*
}

Recibido: agosto 21/2001 - Revisado: octubre 2/2001 - Aceptado: mayo 30/2002

En el 2002 estamos recordando los cincuenta años de la presentación del estudio sobre el popular puntaje Apgar, realizada en el $27^{\circ}$ Congreso Anual de Anestesistas, reunión conjunta de la Sociedad Internacional de Investigación en Anestesia y el Colegio Internacional de Anestesistas, en septiembre de 1952, en Virginia Beach; este análisis sencillo de un sistema fácil y expedito de valorar a un recién nacido, ha resultado de extraordinaria utilidad para anestesiólogos, ginecoobstetras y pediatras neonatólogos (1).

Virginia Apgar (1909-1974) fue ante todo una mujer excepcional. Es cierto que su niñez quedó marcada por las veleidades científicas de su padre, quien incursionó en la astronomía con su telescopio artesanal y se interesaba en la inventiva (experimentado con electricidad y ondas radiales) en un laboratorio que mantenía en el sótano de su casa; hasta llegó a publicar tal cual experiencia sobre el planeta Júpiter. Pero los ingresos en casa de su padre fueron más bien escasos. En cuanto a su relación con los médicos, ésta estuvo determinada por la precoz muerte de su hermano (falleció de tuberculosis a los tres años) o por las frecuentes visitas al doctor de otro de ellos, afectado por un eczema infantil que lo tornó un enfermo crónico. Pero su tenacidad y positivismo se pudieron ver por sus estudios en tiempos de la preguerra, ya que cuando terminó College en Mount Holyoke, podría decirse que era un espécimen raro pues casi ninguna mujer terminaba su bachillerato (2).

Más fuera de lo común era estudiar medicina $\mathrm{y}$, sobre todo, en una escuela tan competitiva como la de Columbia University en Nueva York, para después medírsele a un internado quirúrgico en el Presbyterian, con un jefe de la talla del doctor Alan Whipple, lo que fue ciertamente un logro, aunque con frustraciones.

\footnotetext{
* Internista-endocrinólogo. Miembro de Número de la Academia Nacional de Medicina, de la Sociedad Colombiana de Historia de la Medicina y Honorario de la Asociación Colombiana de Endocrinología.
}

La cirugía era entonces extremadamente competida en la ciudad de Nueva York, y la experiencia de las escasas mujeres cirujanas que se habían entrenado con Whipple no había sido buena. Este profesor consideraba que las intervenciones quirúrgicas estaban tremendamente limitadas por un deficiente proceso anestésico, por lo que se necesitaban profesionales que incursionaran en esta especialidad. Así que, en parte aconsejada por su maestro, y en parte porque la anestesia era como una dependencia de la cirugía -no obstante ser considerada labor de enfermeras-, al poco tiempo de trabajar en la Gran Manzana precisamente con una de estas enfermeras anestesistas, logró vincularse (por seis meses) al Departamento de Anestesiología de la Universidad de Wisconsin, en Madison.

Para encontrar esta posición en una de las trece instituciones americanas que ofrecían entrenamiento en este campo, ella tuvo que escribir al doctor Frank McMechan quien fungía como secretario de "Anestesistas Asociados de Estados Unidos y Canadá", la organización más importante de la especialidad en el país que, insistimos, era básicamente una labor de enfermería. Por dificultades de alojamiento, Apgar dejó este departamento dirigido por Ralph Waters (3) y regresó al Bellevue Hospital de Nueva York, para laborar al lado de Ernest Rovenstine, donde tuvo que dormir en los alojamientos para las muchachas del servicio, lo que por primera le hizo inscribir su queja en el Diario que llevaba.

En 1938 la doctora Virginia obtuvo al fin un reconocimiento al ser nombrada "Directora de la División de Anestesia y Anestesista Adscrita" en su Hospital de Columbia. Era sólo una luz al final del túnel, ya que la carga de trabajo era apabullante, imposible conseguir residentes que ayudaran (la primera residente, Ellen Foot, ingresó dos años más tarde), la compensación económica era inadecuada y los cirujanos, a quienes habría que imaginar como unas "Prima Donna", difícilmente aceptaban que estos médicos al frente de la máscara de los gases, pudieran ser verdaderos colegas. 
La Segunda Guerra Mundial ejerció una influencia definitiva en el ejercicio de la anestesia, pues aunque el trabajo se incrementó notablemente pues los hombres marcharon al frente de batalla, al regreso hubo un incremento de solicitudes para residencia (en 1948 ya tenían dieciocho médicos entrenándose en el programa de Columbia), y la anestesia era ya entonces administrada por más médicos que enfermeras.

En cuanto a los cirujanos, los de más edad se habían acostumbrado a dar ellos mismos el anestésico. Creían pues que tenían la información sobre lo que mejor podía funcionar al paciente, así que la labor de concientización la tuvo que hacer Apgar con los cirujanos jóvenes, que tenían una mentalidad más abierta. En cuanto a los honorarios, un anestesista (como denominaban a los anestesiólogos de la época), no podía cobrar, así que recibían algo de lo que el hospital cobraba por derechos de sala (4).

Podríamos imaginarnos a la acelerada doctora, en su lucha por rescatar su especialidad de la especie de lodo en que se encontraba, con su hablar rápido que describieron unos residentes de anestesia en la fiesta de graduación, con letra adaptada a un ritmo popular de Norteamérica:

"El único consejo que te puedo dar

si es que puedes aprender el truco es que hables tan rápido como yo así que nadie te pueda contestar".

"Aunque algunos dicen que hablo muy rápido, yo les sostengo que están equivocados porque puedo decirles el doble de cosas y me gasto sólo la mitad del tiempo".

Apgar aspiraba a formar un Departamento de Anestesiología -no una simple División- con especialistas médicos y residentes, reduciendo poco a poco el campo a las enfermeras anestesistas, quienes eran pacientes, dedicadas y técnicamente hábiles, característica considerada especial del sexo femenino. Esto lo logró, más no la deseada Dirección del Departamento, quien la ocupó meses más tarde el anestesiólogo del Bellevue, Emmanuel Papper.

Esto llevó a la doctora Virginia a dedicarse a la anestesia obstétrica (5). Estando dedicada a estos menesteres, alguna mañana de 1949, durante un desayuno de trabajo, un estudiante que rotaba por anestesia dijo algo en relación con la necesidad de tener un método de valoración de los recién nacidos.
"Eso es fácil, te mostraré cómo se hace" dijo Apgar, anotando los cinco puntos de lo que sería el famoso método en un pedazo de papel que encontró sobre la mesa. Acto seguido se dirigió a obstetricia, para poner en práctica la idea que había tenido (6-8).

Como recordamos, este método efectivo y fácil de valorar un recién nacido al minuto (como fue inicialmente propuesto por la doctora Apgar) y a los cinco minutos, se basa en puntajes que van de 0 a 2 para cada uno de los parámetros de frecuencia cardiaca, respiraciones, tono muscular, color de la piel y reflejos. En forma resumida, el esquema es como sigue:

Frecuencia cardiaca: ausente (0), < 100 (1), > 100 (2).

Respiraciones: ausentes (0), lentas e irregulares (1), -llanto fuerte y bueno- seguramente en aquellos cincuentas, después de la tradicional nalgada (2).

Tono muscular: flácido (0), alguna flexión de brazos y piernas (1), movimientos activos (2).

Color: completamente azul o pálido (0), azul en manos y pies, pero rosado en el cuerpo (1), completamente rosado (2).

Reflejos: ausentes (0), mueca (1), mueca y tos (ya en las posteriores épocas del succionador "Gomco", inducida por éste) o estornudo (2).

Un tiempo más tarde, alguien se ingenió un acróstico que facilitaría el recuerdo del puntaje. Dice así:

A pariencia (color de la piel)

$\mathbf{P}$ ulso

G rimace (reflejos)

A ctividad (muscular)

$\mathbf{R}$ espiración.

La semilla germinaba, y el interés por la investigación fructificó con la contratación de dos expertos -el anestesiólogo Duncan Holaday y el pediatra Stanley James-, lo que permitió la incorporación de tecnologías para medir el equilibrio ácido-básico y los gases sanguíneos. Pudieron demostrar que los bebés acidóticos e hipóxicos tenían bajos puntajes Apgar (9-11). Por un accidente afortunado pudieron cateterizar la arteria umbilical, y en la inquietud y curiosidad permanentes de la anestesióloga, por medio de un catéter para succionar, pudo desarrollar un método también simple para detectar, al momento del nacimiento, la atresia de las coanas, la fistula tráqueo-esofágica, la atresia duodenal y el ano imperforado. El polihidramnios -como lo publicaría más tarde- estaba asociado con malformaciones congénitas (12). 
James tenía conocimientos de cardiología y estaba interesado en lograr el desarrollo de métodos de resucitación cardiaca en recién nacidos; en efecto lo logró, demostrando que el sistema de oxígeno intragástrico utilizado hasta aquel momento era inefectivo, popularizando el uso del laringoscopio y de la intubación, y preparando una película sobre técnicas de resucitación que tuvo amplia difusión gracias al apoyo económico de un laboratorio farmacéutico.

Los aportes de Holaday fueron varios, entre ellos la descripción de una técnica de depuración de nitrógeno para medir el ciclopropano, la utilización del microgasómetro de Nadelson para medir gases arteriales en la presencia de agentes anestésicos, y antes de comprar uno de los primeros electrodos de Astrup para medir $\mathrm{pH}$ fácilmente, logró métodos para determinarlo de una mejor forma (5).

El grupo demostró que el ciclopropano era particularmente depresor para el recién nacido, con lo que este popular gas perdió todo papel en anestesia obstétrica. Curiosamente era el agente preferido por Apgar, pues creía que era completamente seguro e inocuo. Pero cuando sus asociados le demostraron que esto no era así, de inmediato lo aceptó y a la hora del almuerzo dio la despedida a su gas favorito (10).

Entre 1938 y 1946, el departamento incorporó todas las nuevas tecnologías y descartó otras como el uso obstétrico del "ciclo". Se empezaron a usar el óxido nitroso, el tiopental, el curare, el bloqueo nervioso, el uso de caucho conductivo y después el no conductivo, etc. Dentro de sus residentes egresados, dos de ellos, Frank Moya y Sol Schneider, llegaron a ser grandes investigadores en anestesia obstétrica.

El trabajo original sobre el puntaje Apgar se publicó en 1953 (13); años después se desarrolló un

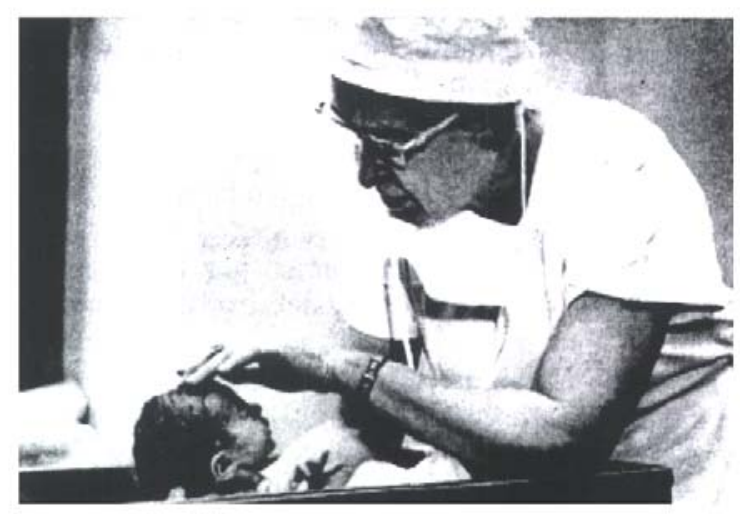

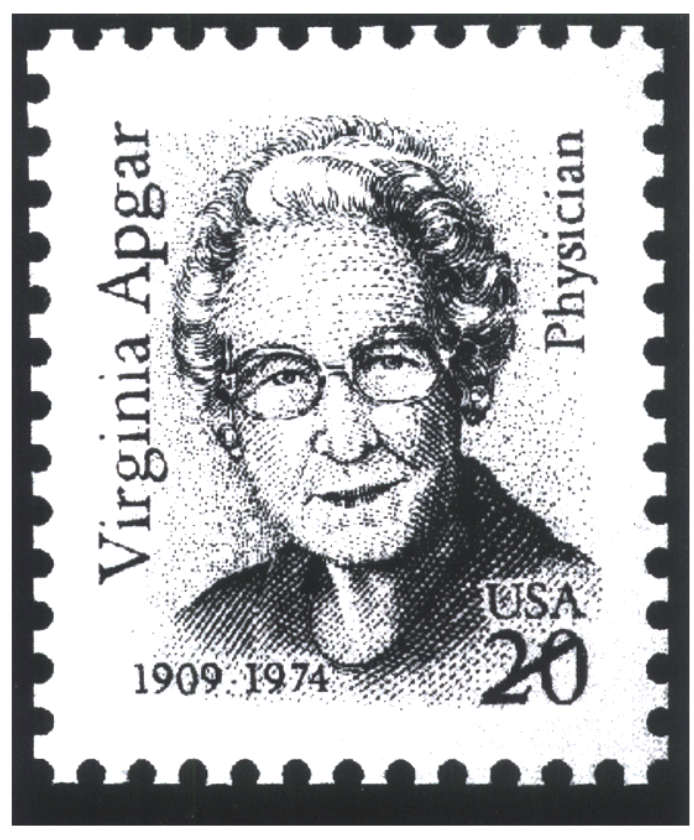

estudio conjunto en doce instituciones, en el que se demostró, en un total de 17.221 bebés, que el puntaje Apgar - y en particular el realizado a los cinco minutos-, era un predictor de supervivencia neonatal y del desarrollo neurológico futuro (14).

Entre 1960 y 1974, cuando falleció víctima de un cáncer, estuvo como directora de la división de malformaciones congénitas de la Fundación Nacional, la que ha sido más conocida como "La Marcha de las Monedas". Previamente había realizado una maestría en Salud Pública en la Universidad Johns Hopkins, con el fin de mejorar sus conocimientos sobre estadística.

No todo en la vida de Virginia Apgar fue medicina ni anestesiología. Le encantaba pescar, trabajar en el jardín y seguir los juegos de béisbol de las Grandes Ligas. Era filatélica consumada, y aprovechaba sus viajes para coleccionar estampillas de numerosos países; perteneció a la Sociedad Americana de Filatelia, y ella misma fue objeto de un estampilla recordatoria (15). Un tiempo después de su muerte ingresó al Salón de la Fama de las Mujeres, al lado de otras importantes damas que descollaron en campos muy difíciles, antes vedados a personas del sexo femenino (16-17).

Apgar fue devota de la música clásica, y participó como violinista en cuartetos integrados por médicos; además aprendió a fabricar instrumentos de cuerda y cuatro de ellos se exhiben en una sala de exposiciones.

Tan sólo por sus aficiones no médicas podríamos decir que su vida fue extraordinaria, pues en ellas se 
desempeñó con maestría y entusiasmo. No era persona de perder el tiempo ni de hacer las cosas a medias. Hizo numerosas amistades entre colegas, alumnos y pacientes. En alguna oportunidad, una médica, antigua alumna suya, le pidió que la asistiera con los gases en el momento de dar a luz; le fue imposible llegar a tiempo, pero habiendo nacido el bebé, se dedicó a tomar fotografías del acontecimiento (2).

Fue pionera en anestesia obstétrica y dio las bases de la perinatología. No sólo es recordada por los anestesiólogos, sino por los pediatras neonatólogos y particularmente por los obstetras en su labor cotidiana.

\section{REFERENCIAS BIBLIOGRÁFICAS}

1. Steele R. Apgar Scoring, what is it? www.parentsplace.com/

2. Enocha BE. Virginia Apgar, a legend becomes a postage stamp. P\&S Journal 1994;14 (3).

3. Waters RM. Anesthesiology in the hospital and in the medical school. JAMA 1946; 130 : 909-912.

4. Calmes SH. Virginia Apgar, a woman physician's career in a developing specialty. J Am Med Women's Assoc 1984; 39: 184188.

5. Calmes SH. Virginia Apgar, MD, At the Forefront of Obstetric Anesthesia. ASA. Newsletter Oct. 1992; 9-12.

6. Calmes SH. Development of the Apgar Score. In: Anaesthesia, Essays on lts History. Ruphret J, et al. (Eds). Springer-Verlag, Berlin, 1985.

7. Lemelson-MIT Prize Program. Virginia Apgar, Newbom Scoring System. Inventor of the Week Archives. www.web.mit.edu/

8. Calmes SH. And what about the baby? Virginia Apgar and the Apgar Score. ASA Newsletter Sept. 1997; 20-22.

9. Apgar V, Holaday DA, James LS, Weisbrot IM. Evaluation of the newborn infant, second report. JAMA 1958; 165: 1985-88.

10. Apgar V, Holaday DA, James LS. Comparison of regional and general anesthesia in obstetrics. JAMA 1957; 165: 2155-2161.

11. Apgar V. The newborn (Apgar) scoring system. Pediat Clin N Am 1966; 13: 645650.

12. Moya F, Apgar V, James LS, Berien C. Hydramnios and congenital abnormalities. JAMA 1960; 173: 1552-1556.
13. Apgar V. A proposal for a new method of evaluation of the newborn infant. Curr Res Anesth Analg 1953; 32: 260-267.

14. Drage JS, Kennedy C, Schwartz BK. The Apgar Score as an index of neonatal mortality. Obstet Gynecol 1964; 24: 222.

15. US Postal Service. New Stamp scores a "10" on the Apgar Scale. Stamps News Release \# 94-041. www.apgar.net/

16. Apgar E. Speech on behalf of the Apgar family.

17. Calmes SH. Virginia Apgar, MD, Inducted into National Women's Hall of Fame. ASA Newsletter 1995; 59 (12). 